\title{
Evaluación de la cobertura de la merienda escolar en 4 departamentos de Paraguay. Años 2016-2018
}

\author{
Evaluation of school meal coverage in 4 departments of \\ Paraguay. During 2016-2018
}

\author{
Laura Mendoza de Arbo', Susana Sánchez Bernal'
}

\section{RESUMEN}

Introducción: La merienda escolar contribuye con la nutrición y rendimiento escolar, es importante conocer su alcance. Objetivo: Evaluar la merienda escolar en Concepción, Cordillera, Paraguarí y Central, en cuanto implementación y cobertura. Materiales y Métodos: Estudio observacional descriptivo retrospectivo de licitaciones para merienda escolar entre 2016-2018 en Concepción, Cordillera, Paraguarí y Central. Fueron caracterizadas las instituciones incluidas, días cubiertos del calendario escolar (180 días) y cantidad de beneficiarios. Resultados: Fueron incluidas 15 licitaciones. En Concepción 90\% fueron instituciones públicas, $6 \%$ privadas, $3 \%$ sin datos; Cordillera $88 \%$ fueron públicas, $8 \%$ privadas, $4 \%$ sin datos; Paraguarí $91 \%$ fueron públicas, $3 \%$ privadas, resto sin datos; Central $12 \%$ fueron privadas $11 \%$ sin datos, resto públicas. En Concepción fueron 30.112 beneficiarios en 2016/2017 y 27.514 en 2018, en Cordillera aumentó de 37.167 (2017) a 44.375 (2018); en Paraguarí varió de 30.339 (2016) a 29.761 (2018), en Central fueron 180.535 beneficiarios en 2017-2018. En Concepción en 2016/2017 se planificó merienda para 80 días, 133 días en 2018, en Cordillera 160 días en 2017, 117 días en 2018; en Paraguarí en 2016 fueron 122 días, 110 en 2017 y 117 días en 2018; en Central fueron 216 días en 2017-2018.Los beneficiarios cubiertos según Registro Único de Estudiantes al 2018 fue 78,5\% en Concepción, 87\% en Cordillera, 90\% en Paraguarí, $75 \%$ en Central. Conclusiones: En provisión de merienda escolar, la mayoría fueron instituciones públicas, hubo descenso de beneficiarios en el 2018 excepto Cordillera. 2/3 de los beneficiarios registrados fueron incluidos y se proveyó durante poco más de la mitad del año escolar.

Palabras claves: Merienda escolar, estudiantes, instituciones educativas, beneficiarios.

\section{ABSTRAC}

Introduction: The school meal contributes to nutrition and school performance, it is important to know its reach. Objective: To evaluate school meals in Concepción, Cordillera, Paraguarí and Central, in terms of implementation and reach. Materials and Methods: This was a retrospective, descriptive, observational study of awarded contracts for school meals between 2016-2018 in Concepción, Cordillera, Paraguarí and Central. We characterized the included institutions, covered days of the school calendar (180 days) and number of beneficiaries. Results: 15 contracts were included. In Concepción, $90 \%$ of schools were public institutions, $6 \%$ private, $3 \%$ without data; in Cordillera $88 \%$ were public, $8 \%$ private, $4 \%$ without data; in Paraguarí $91 \%$ were public, $3 \%$ private, the rest without data; in Central $12 \%$ were private, $11 \%$ without data, the rest public. In Concepción there were 30,112 beneficiaries in 2016/2017 and 27,514 in 2018, in Cordillera it increased from 37,167 (2017) to 44,375 (2018); in Paraguarí it varied from 30,339 (2016) to 29,761 (2018), in Central there were 180,535 beneficiaries in 2017-2018. In Concepción in 2016/2017 meals were planned for 80 days, 133 days in 2018, in Cordillera 160 days in 2017, 117 days in 2018; in Paraguarí in 2016 there were 122 days, 110 in 2017 and 117 days in 2018; In Central there were 216 days in 2017-2018. The beneficiaries covered according to the Single Student Registry in 2018 were $78.5 \%$ in Concepción, $87 \%$ in Cordillera, 90\% in Paraguarí, 75\% in Central. Conclusions: In the provision of school meals, the majority of schools were public institutions, there was a decrease in beneficiaries in 2018 except in Cordillera. 2/3 of the registered beneficiaries were included and meals were provided for just over half the school year.

Keywords: School lunch, students, educational institutions, beneficiaries.

\footnotetext{
${ }^{1}$ Salud y Nutrición Paraguay (SANUPAR). Fernando de la Mora, Paraguay.

Correspondencia: Susana Sánchez Bernal Correo: susana.sanchez.ber@gmail.com

Recibido: 09/09/2020 Aceptado:25/09/2020

Conflicto de interés: Los autores declaran no poseer conflicto de interés.

Doi: https://doi.org/10.31698/ped.47032020006
} 


\section{INTRODUCCIÓN}

La alimentación es un derecho humano universal ${ }^{(1)}$ cuyo cumplimiento corresponde garantizar al Estado ya que estima que el hambre afecta a millones de personas en el mundo ${ }^{(2,3)}$.

El niño es especialmente susceptible al hambre ya que afecta el desarrollo pleno de su potencial. En la edad escolar, el no recibir los nutrientes necesarios perturba su capacidad de aprendizaje aumentando la probabilidad de repitencia o deserción escolar y compromete su productividad ${ }^{(4-6)}$ en lo cual se sustenta la importancia de provisión de alimentos a los niños en edad escolar en todo el mundo pues su impacto es notorio en el rendimiento escolar ${ }^{(7)}$.

Desde hace más de dos décadas en el Paraguay, bajo diferentes denominaciones programáticas se realiza la entrega de un complemento nutricional en instituciones educativas, para colaborar en el aspecto nutricional de los estudiantes y a la par, lograr una mejor matriculación, una menor deserción escolar y mejorar el promedio de escolaridad. El desafío prevenir desnutrición o el hambre oculta y no no propiciar el exceso de peso, ya que nuestro país no está exento de esta realidad ${ }^{(8)}$.

Según la Ley $\mathrm{N}^{\circ} 5210$ “De la Alimentación Escolar y Control Sanitario" promulgada en el 2014, el objetivo es "garantizar el bienestar físico del estudiante durante el período de asistencia en la Institución Educativa", en ella también se establece que "el Gobierno Central, Departamental y Municipal, deberán desarrollar e implementar estrategias de monitoreo y evaluación de procesos, y de impacto de la implementación de la alimentación escolar ${ }^{\prime{ }^{\prime(9)}}$.

El actual Programa de Alimentación Escolar del Paraguay (PAEP) fue creado en el año 2015, como parte de una política de protección social que contempla la entrega directa de "raciones alimenticias inocuas, nutritivas y culturalmente aceptadas a los niños matriculados en el sistema educativo tanto público como subvencionado ${ }^{\prime(10,11)}$ lo cual se traduce en la provisión de merienda y de almuerzo escolar.

Si bien el Ministerio de Educación y Ciencias (MEC) es responsable del PAEP, su implementación es descentralizada ya que éste se ocupa de la capital mientras que en el resto del país desde gobiernos departamentales y depende de su capacidad económica y de gestión. Muy poco se sabe de las diferencias de ejecución del programa entre los diferentes gobiernos departamentales.

Por lo mencionado antes, el objetivo de esta investigación fue evaluar el PAEP en su componente de merienda escolar en los departamentos de Concepción, Cordillera, Paraguarí y Central, en cuanto a las instituciones incluidas, duración de la provisión de la merienda según calendario escolar y cobertura de beneficiarios.

\section{MATERIALES Y MÉTODOS}

Estudio retrospectivo, descriptivo, observacional de corte transversal en base a datos secundarios obtenidos de la Dirección Nacional de Contrataciones Públicas (DNCP) y de los Datos abiertos del Ministerio de Educación y Ciencias MEC).

El muestreo fue no probabilístico de casos consecutivos que incluyó todos los procesos licitatorios de los departamentos de Concepción, Cordillera, Paraguarí y Central realizadas durante los años 2016, 2017 y 2018 disponibles en las fuentes antes citadas. Todas las licitaciones tanto el pliego de bases y condiciones (PBC), contratos, documento de adjudicación, entre otros) con sus respectivos documentos adjuntos fueron descargadas de la página de la DNCP y los datos relacionados con las variables estudiadas, fueron sistematizadas en una planilla electrónica por las investigadoras.

Fueron evaluadas las siguientes variables: cantidad y características de las instituciones según tipo de gestión, el tipo de complemento nutricional adquirido ya sea leche o el complemento sólido (galletitas, cereales, entre otros) cantidad total de estudiantes beneficiados por año. También fue determinada la cantidad de días para entrega del complemento nutricional según lo programado en el PBC versus el calendario escolar anual que corresponde a unos 180 días en promedio. 
A efectos de valorar el alcance de beneficiarios de la merienda escolar, se realizó el análisis de la cobertura de niños que deberían recibirla conforme a lo establecido en los PBC de los diferentes procesos licitatorios comparando con los inscriptos en el Registro Único del Estudiante (RUE) a partir de lo cual se pudo calcular el porcentaje de beneficiarios según la siguiente fórmula: $\%$ cobertura $=\left(\mathrm{n}^{\circ}\right.$ beneficiarios $/ \mathrm{n}^{\circ}$ inscriptos RUE)x100.

Cabe destacar que el RUE fue puesto en marcha en el 2017, alcanzando el registro real en el 2018, por lo cual fueron utilizados estos datos como referencia para el cálculo antes presentado para el cálculo de la cobertura.

Dada la naturaleza de estudios y los datos utilizados no se requirió la aprobación de un Comité de Ética ya que no se trabajó con datos de personas. En todo momento se garantizó la confidencialidad de los datos, incluyendo los nombres de las instituciones participantes sujetos e incluso la identidad de las instituciones educativas involucradas fueron preservadas.
Procesamiento y análisis de los datos fue realizado con la planilla electrónica Microsoft Excel y con el paquete estadístico SPPS versión 20. Los datos se presentan en frecuencia y porcentajes.

\section{RESULTADOS}

Fueron incluidas 15 licitaciones adjudicadas en los departamentos de Concepción, Cordillera, Paraguarí y Central para la provisión de merienda escolar entre los años 2016 al 2018. Cabe aclarar que en el Departamento Central se incluyó un solo proceso licitatorio plurianual para los años 20172018, ya que el año 2016 fue cubierto por la licitación del año previo.

En el Gráfico 1 se muestran los porcentajes de instituciones según tipo de gestión (oficial, subvencionada o privada) que fueron incluidas como beneficiarias. En el Departamento Central se observó un $11 \%$ de instituciones que no pudieron ser identificadas y $12 \%$ de instituciones de gestión privada o privada subvencionada.

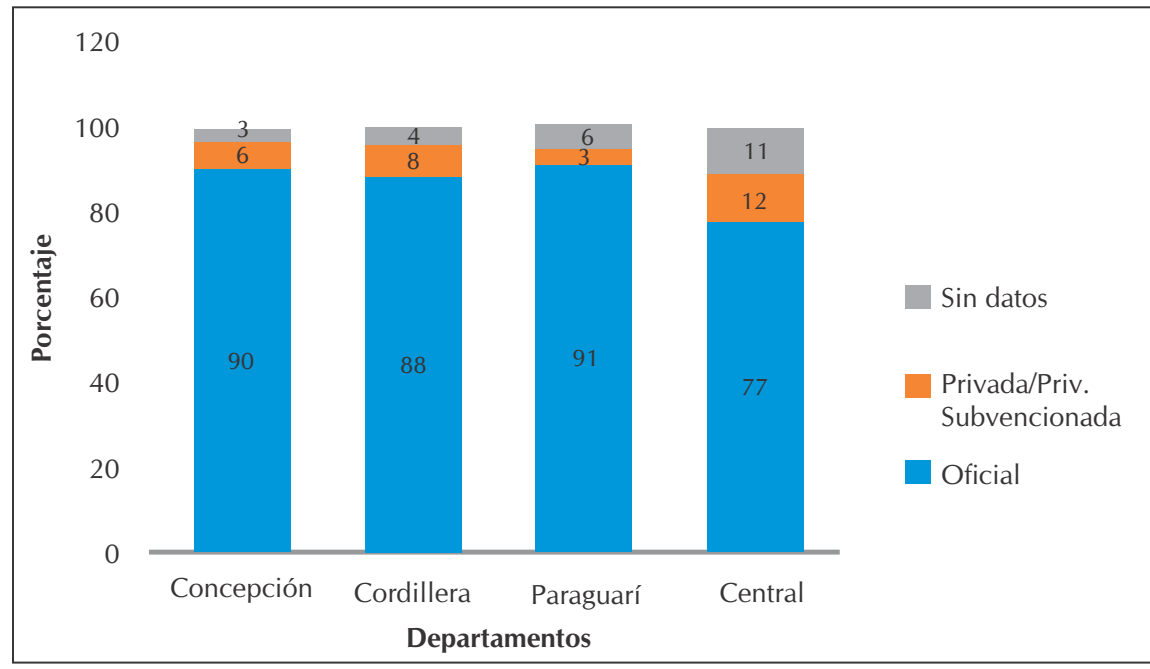

Gráfico 1. Porcentaje de instituciones beneficiadas con la merienda escolar según tipo de gestión en los Departamentos de Concepción, Cordillera, Paraguarí y Central. Años 2016- 2018.

Fueron analizados por departamento y por año, las licitaciones el monto adjudicado, el número de instituciones y de estudiantes beneficiarios y los dias totales de cobertura. Puede notarse que la mayoría de las adquisiciones fueron leche fluida y galletitas (Tabla 1). El número de estudiantes disminuyó en un 8,6\% entre 2016 y 2018 en Concepción, aumentó en 16,2\% en Cordillera, bajó en 10,3\% en Paraguarí. 
Tabla 1. Licitaciones por año, proveedores, montos adjudicados, productos adquiridos, instituciones y cantidad de beneficiarios. Gobernaciones de Concepción, Cordillera, Paraguarí y Central.

\begin{tabular}{|c|c|c|c|c|c|c|}
\hline Departamento & Año de licitación & $\begin{array}{l}\text { Monto Adjudicado } \\
\text { (Guaraníes) }\end{array}$ & $\begin{array}{l}\text { Productos } \\
\text { adquiridos }\end{array}$ & $\begin{array}{c}\text { Instituciones } \\
\text { Beneficiadas N }\end{array}$ & \begin{tabular}{|c|} 
Estudiantes \\
beneficiarios $\mathbf{n}$
\end{tabular} & \begin{tabular}{|c} 
Días cubiertos \\
$\mathrm{N}$ \\
\end{tabular} \\
\hline \multirow{4}{*}{ Concepción } & 2016-2017 & 8.272 .236 .800 & Leche fluida & 382 & 30.112 & 80 \\
\hline & 2016-2017 & 6.211 .872 .704 & $\begin{array}{l}\text { Galletita cracker, } \\
\text { galletita dulce, } \\
\text { galletita con chía } \\
\text { o sésamo }\end{array}$ & 382 & 30.112 & 80 \\
\hline & 2018 & 2.550 .016 .280 & $\begin{array}{l}\text { Galletita cracker, } \\
\text { galletita dulce, } \\
\text { galletita con chía } \\
\text { o sésamo }\end{array}$ & 362 & 27.514 & 133 \\
\hline & 2018 & 3.468 .559 .250 & Leche fluida & 362 & 27.514 & 133 \\
\hline \multirow{3}{*}{ Cordillera } & 2017 & 7.052 .809 .920 & Leche fluida & 338 & 37.167 & 160 \\
\hline & 2018 & 3.953 .907 .400 & Leche fluida & 383 & 44.375 & \multirow[t]{2}{*}{117} \\
\hline & 2018 & 1.360 .850 .400 & Leche fluida & 385 & 44.375 & \\
\hline \multirow{5}{*}{ Paraguarí } & 2016 & 3.790 .960 .640 & Leche & 385 & 30.339 & \multirow[t]{2}{*}{122} \\
\hline & 2016 & 2.540 .891 .250 & Alimento sólido & 385 & 30.339 & \\
\hline & 2017 & 2.300 .520 .684 & Galletitas & 387 & 30.968 & \multirow[t]{2}{*}{110} \\
\hline & 2017 & 3.761 .689 .932 & Leche fluida & 387 & 30.968 & \\
\hline & 2018 & 5.993 .089 .290 & $\begin{array}{l}\text { Leche fluida, Palito, } \\
\text { alimento a base de } \\
\text { mani, cereal }\end{array}$ & 383 & 27.761 & 117 \\
\hline \multirow[b]{3}{*}{ Central } & 2017 & 21.639 .106 .656 & Leche fluida & 715 & 180.535 & \multirow[t]{3}{*}{126} \\
\hline & 2018 & 21.701 .011 .984 & Leche fluida & 715 & 180.535 & \\
\hline & $2017-2018$ & 51.078 .171 .350 & $\begin{array}{l}\text { Cereal } \\
\text { Pan Lactal } \\
\text { Crackers }\end{array}$ & 715 & 180.535 & \\
\hline
\end{tabular}

También en la Tabla 1 se muestran los días que estaban planificados la provisión de merienda escolar.

En el Gráfico 2 se pueden observar los porcentajes de días que según los documentos analizados, fueron cubiertos con las licitaciones adjudicadas por departamento y por año con respecto al total de los 180 días previstos para el calendario escolar oficial y se destaca que en Concepción este porcentaje fue de 44\% en el 2016-2017 mientras que en Cordillera para ese mismo tiempo fue de $89 \%$ y del $65 \%$ en el siguiente año en el que también se registró un aumento de beneficiarios en este departamento. Paraguarí por su parte presentó coberturas similares en los 3 años, y Central según registro documental debería haber cubierto el $70 \%$ de días del año escolar.

En cuanto a la cobertura de beneficiarios, se evaluó la proporción de beneficiarios que serían alcanzados según lo programado en los procesos licitatorios versus los estudiantes oficialmente registrados en el RUE de las instituciones educativas incluidas, así como también se analizó el porcentaje de cobertura considerando la totalidad de los estudiantes de cada departamento estudiado durante los años de estudio. Estos datos se presentan en el Gráfico 3, en el cual se observa que ajustando por RUE Paraguarí mostró una cobertura del 90\% según lo programado mientras que en el Departamento Central fue de $75 \%$.

En este contexto, al analizar el alcance global del programa en cuanto al porcentaje de la población estudiantil total registrada por RUE, se observó que la cobertura departamental con la merienda escolar fue del $87 \%$ en el departamento de Cordillera y del $52 \%$ en el departamento Central (Gráfico 3). 


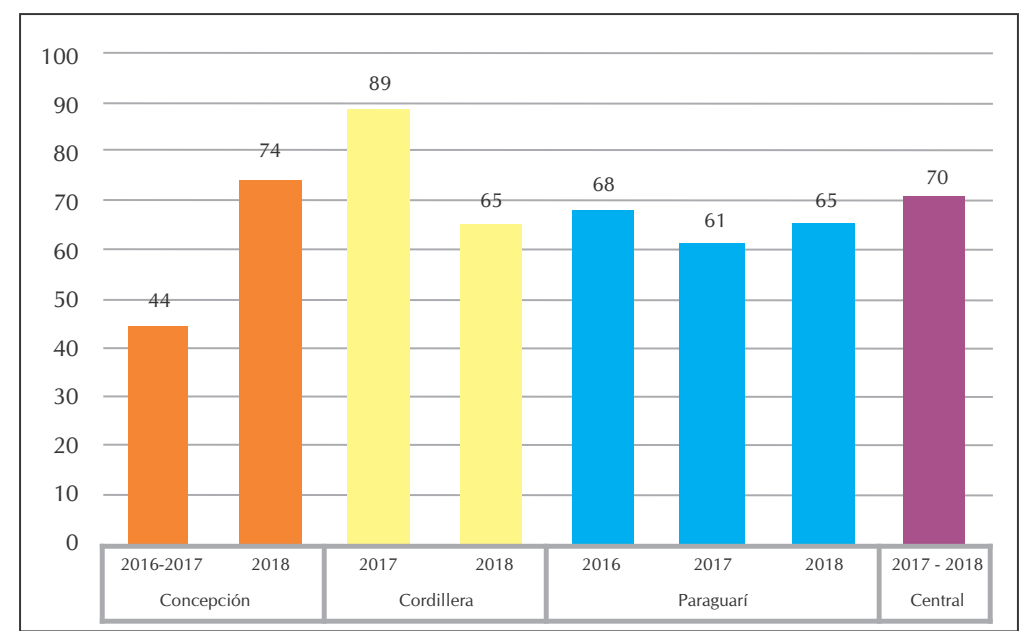

Gráfico 2. Porcentaje de días cubiertos de merienda escolar en base al calendario escolar en los departamentos de Concepción, Cordillera, Paraguarí y Central. Años 2016-2018.

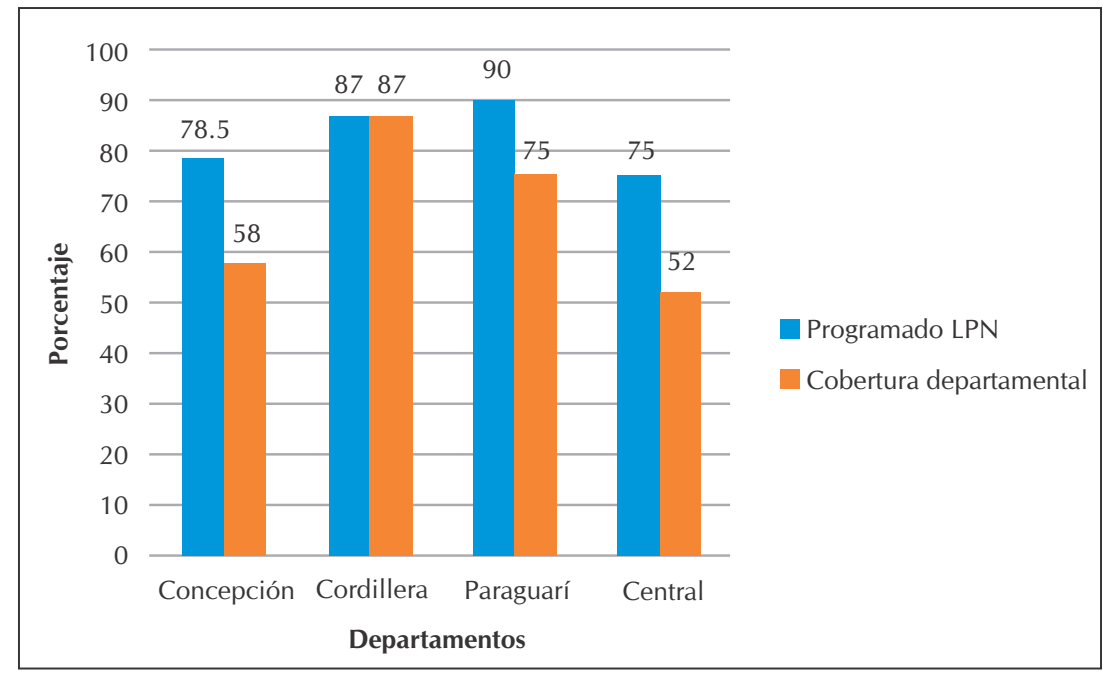

Gráfico 3. Comparación de cobertura de estudiantes beneficiados programados en licitaciones y cobertura departamental en porcentajes según RUE.

\section{DISCUSIÓN}

En este estudio se muestra el presupuesto destinado a merienda escolar en cuatro departamentos del Paraguay, así como el alcance en término de cobertura de beneficiarios y de días de provisión del complemento nutricional.

En este trabajo se ha encontrado que, en ninguno de los departamentos evaluados, el PAEP logra la cobertura deseada en cuanto a la provisión de la merienda escolar.
El presupuesto que reciben las Gobernaciones de los departamentos estudiados que utilizan para suministrar tanto la merienda como el almuerzo escolar es insuficiente para cubrir ambas modalidades, por lo cual no suministran ninguna de las dos prestaciones en forma efectiva ni durante todo el año escolar por lo cual no cumplen con el fin para el cual fueron creados, incrementando inequidades por lo que urge la reestructuración programática y presupuestaria. Es importante comprender que la ejecución de cualquier programa 
debe ser eficiente y eficaz, a fin de demostrar su impacto y justificar su sostenibilidad en el tiempo(12).

Imas y Ávila ${ }^{(13)}$, señalan que la distribución de los recursos no solo es inequitativa, sino que al mismo tiempo es reproductora de la desigualdad territorial. Las Gobernaciones y Municipalidades no afectadas por las represas hidroeléctricas, reciben en promedio menos recursos por estudiante que los Gobiernos locales afectados, y es entre los territorios no afectados donde se encuentran los departamentos con mayor incidencia de pobreza tanto total como extrema y con mayores necesidades de inversión en infraestructura.

Si bien está estipulado que parte de los alimentos destinados a la alimentación escolar provengan de la agricultura familiar, poco se sabe de la inversión en las compras públicas para tal efecto ${ }^{(14)}$ puesto que, si bien todas las licitaciones evaluadas en este estudio incluyeron la provisión de leche fluida y como complemento sólido, se adquirió principalmente galletitas.

La presente investigación está basada en datos secundarios disponibles en formato digital y en la web de las instituciones descritas en la parte metodológica y la veracidad depende de la información incluida en los documentos a los que las investigadoras pudieron acceder. Por lo expuesto, como limitación, este documento contiene información de declaraciones, pero no de verificación in situ de su llegada al beneficiario final que es el estudiante, ni tampoco evalúa el tiempo y forma de la provisión de insumos del PAEP, etapas extremadamente importantes para poder llevar a nuestros niños alimentos de calidad e inocuos y que complementan los principios universales que rigen al PAEP.

Es fundamental contar con un sistema regular de monitoreo y evaluación a fin de evaluar la calidad de los productos, su aceptabilidad y consumo ${ }^{(15)}$.

En nuestro medio el estudio realizado por Sanabria et al. mostró que sólo a 3 de cada 10 niños les agradan las comidas que les sirven y que la adecuación de energía y proteínas no se ajustan a los requerimientos recomendado ${ }^{(16)}$.
Como limitante por la naturaleza del trabajo, tampoco se pudo analizar, la adecuación en calidad y cantidad del complemento nutricional, lo cual es importante evaluarlo a futuro ya que en la actualidad un efecto negativo de cualquier programa sería contribuir al aumento del exceso de peso ${ }^{(17)}$ por lo tanto a pesar de la perspectiva de derecho a la alimentación, se plantean varios desafíos concernientes a este tipo de políticas públicas ${ }^{(18)}$.

Es posible también que sea tiempo de replantear el modelo de implementación actual del PAEP que implica una actitud pasiva de los estudiantes y sus familia y escalar a modalidades que involucren a cuidadores y al propio niño aún en situación de pobreza ${ }^{(19)}$ tal como se ha visto en otros países en los que mejoran no solo los indicadores nutricionales sino la retención escolar y el sentido de responsabilidad ante la autoalimentación ${ }^{(20,21)}$ además del redireccionamiento del enfoque educativo y participativo para mejoría la calidad de la dieta de esta población ${ }^{(22,23)}$.

Es necesario evaluar la reestructuración del PAEP, mejorar la eficiencia del gasto público en el programa e instalar un sistema de monitoreo y evaluación periódica.

\section{CONCLUSIONES}

Según los procesos licitatorios evaluados la mayoría de las instituciones beneficiadas son públicas.

En todos los departamentos hubo descenso de beneficiarios en el 2018 excepto en el departamento de Cordillera.

En promedio se cubre poco más de la mitad del año escolar. Concepción aumentó la proporción de días cubiertos, Cordillera disminuyó, Paraguarí y Central se mantuvieron estables.

La cobertura en cantidad de beneficiarios cubiertos respecto al RUE fue del 2/3 siendo Paraguarí el de mejor alcance. 


\section{REFERENCIAS}

1. ONU. Universal Declaration of Human Rights [Internet]. Paris: ONU; 1948 [Citado 27 abr. 2020]. Paris. Disponible en: http://www.un.org/en/universaldeclaration-human-rights/

2. ONU. Observación general № 12 : El derecho a una alimentación adecuada (artículo 11 del Pacto Internacional de Derechos Económicos, Sociales y Culturales) [Internet]. ONU; 1999 [Citado 27 abr. 2020]. Disponible en: https://www.refworld.org.es/docid/47ebcce12.html

3. FAO, FIDA, OMS, PMA, UNICEF. El estado de la seguridad alimentaria y la nutrición en el mundo 2019: Protegerse frente a la desaceleración y el debilitamiento de la economía [Internet]. Roma: FAO; 2019 [Citado 30 abr. 2020]. Disponible en: http://www.fao.org/3/ca5162es/ca5162es.pdf

4. UNICEF. Estado Mundial de la Infancia 2019: Niños, alimentos y nutrición [Internet]. Nueva York: UNICEF; 2019 [Citado 30 abr. 2020]. Disponible en: https://www.unicef.org/media/62486/file/Estadomundial-de-la-infancia-2019.pdf

5. De la Cruz Sánchez EE. La educación alimentaria y nutricional en el contexto de la educación inicial. Paradigma [Internet]. 2015 [Citado 16 abr. 2020];36(1): 161-183. Disponible en: http://ve.scielo.org/scielo.php?script=sci_ar ttext\&pid=S1011-22512015000100009\&lng=es\&tlng=es

6. Olivera SM. Efectos del desayuno escolar en la tasa de retención del sistema escolar público. Revista Latinoamericana de Desarrollo Económico [Internet]. 2004 [Citado 16 abr. 2020]; (3):77-116. Disponible en: http://www.scielo.org.bo/scielo.php?script=sci_arttext\& pid=S2074-47062004000200004\&lng=es\&tlng=es

7. MacDonald F. Evaluation of the School Breakfast Clubs Program: Final report [Internet]. Australia: Victoria University; 2019 [Citado 16 abr. 2020]. Disponible en: https://www.vu.edu.au/sites/default/files/evaluationschool-breakfast-clubs.pdf

8. Morínigo Ilsa G, Sánchez Bernal S, Sispanov Pankow V, Brizuela Rivarola M, Rolón Villalba G, Mendoza de Arbo L. Perfil Nutricional de Escolares y Adolescentes en escuelas públicas y privadas, 2013. Pediatr. (Asunción). 2015; 42(2):129-133. doi: http://dx.doi.org/10.18004/ped.20 15.agosto.129-133

9. Ley De Alimentación Escolar y Control Sanitario de 2014. Ley N ${ }^{\circ}$ 5510/2014, Congreso Nacional. 2014 [Citado 16 abr. 2020] Disponible en: https://mec.gov.py/ cms_v4/documentos/ver_documento/?titulo=Ley $\% 20 \mathrm{~N} \%$ C2\%BA\%205210/2014
10. Unidad Técnica del Gabinete Social, Ministerio del Ministerio de Educación y Ciencias. Programa de alimentación escolar del Paraguay: evaluación final [Internet]. Paraguay: UTGS, MEC; 2018 [Citado 28 abr. 2020]. Disponible en: https://www.gabinetesocial. gov.py/archivos/documentos/Evaluacion-PAEPFINAL_qr9vai7o.pdf

11. Ministerio de Hacienda. Evaluación de programas públicos: evaluación del diseño y gestión de la provisión de alimento escolar [Internet]. Paraguay: Ministerio de Hacienda; 2017 [Citado 28 abr. 2020]. Disponible en: h t t p s:// w w w. haciend a.gov.py/we bpresupuesto/archivo.php?a=f9f9fck3kek7kdk8kcc7cac8c9 d0c7k9k9kbc7cac8c9cfc5fdkff9k5kef9fbk2k8k7b8f9k5k2k 6fdk7kdk8b8fdkcfbk8k5f9kbc5k6fdfbc5ffk8fafdkbk7f9fb k2k8k7fdkcc6k9fcfef9098\&x=k4k40a2\&y=keke0ac

12. Oostindjer M, Aschemann-Witzel J, Wang Q, Skuland SE, Egelandsdal B, Amdam GV, et al. Are school meals a viable and sustainable tool to improve the healthiness and sustainability of children's diet and food consumption? A cross-national comparative perspective. Crit Rev Food Sci Nutr. 2017;57(18):3942-3958. doi: http://dx.doi.org/ 10.1080/10408398.2016.1197180

13. Imas V, Ávila C. Equidad, gestión y participación en el Fondo Nacional de Inversión Pública y Desarrollo (FONACIDE). Novapolis [Internet]. 2017[Citado 28 abr. 2020];11: 33-53. Disponible en: http://pyglobal.com/ojs /index.php/novapolis/article/view/77/84

14. Rojas J. Las compras públicas de la agricultura familiar en Paraguay. Economía y Sociedad, Análisis de Coyuntura. 2018; 58:17-19.

15. Tugault-Lafleur CN, Black JL, Barr SI. A systematic review of methods to assess children's diets in the school context. American Society for Nutrition. Adv Nutr. 2017;8:63-79. doi: http://dx.doi.org/10.3945/an.116.013144

16. Sanabria M, Frutos D, Preda J, Gonzalez L, Colmelli P. Adecuación y aceptación de almuerzos escolares en dos escuelas públicas de Asunción. Pediatr. (Asunción). 2017;44(2):126-135. doi: http://dx.doi.org/10.18004/ped .2017.agosto.126-135

17. Machado K, Gil P, Ramos I, Pírez C. Sobrepeso/ obesidad en niños en edad escolar y sus factores de riesgo. Arch. Pediatr. Urug. 2018; 89(S1):S16-S25. doi: http://dx.doi.org/10.31134/ap.89.s1.2 
18. Lomaglio D. Retos y desafíos de los programas de nutrición escolar en América Latina Y el Caribe: El caso de Argentina. Arch Latinoam Nutr [Internet]. 2015 [Citado 30 abr. 2020];65(S1):134. Disponible en: https://www.alan revista.org/ediciones/2015/suplemento-1/art-178/

19. Overcash F, Ritter A, Mann T, Mykerezi E, Redden J, Rendahl A, et al. Impacts of a Vegetable Cooking Skills Program Among Low-Income Parents and Children. J Nutr Educ Behav. 2018;50(8):795-802. doi: http://dx.doi.org/10.1016/j.jneb.2017.10.016.

20. FAO, WFP. Home-Grown School Feeding: Resource Framework [Internet]. Roma: FAO; 2018 [Citado 30 abr. 2020]. Disponible en: http://www.fao.org/3/ca0957en/ CA0957EN.pdf
21. Maiz E, Urdaneta E, Allirot X. The importance of involving boys and girls in food preparation. .Nutr Hosp. 2018;35(4):136-139.doi: http://dx.doi.org/10.20960/nh.2139

22. Prescott MP, Lohse B, Mitchell DC, Cunningham-Sabo L. Child assessments of vegetable preferences and cooking self-efficacy show predictive validity with targeted diet quality measures. BMC Nutr. 2019; 2:5:21. doi: http://dx.doi.org/10.1186/s40795-019-0286-7

23. Li PP, Mackey G, Callender C, Dave JM, Olvera N, Alford S, et al. Culinary Education Programs for Children in Low-Income Households: A Scoping Review. Children (Basel). 2020;13;7(5):47. doi: http://dx.doi.org/10.3390/chil dren7050047 